\title{
Determination of heavy metal spallation reactions cross sections at 2, 2.94, 3.5 GeV deuteron beams
}

\author{
Volha Bukhal $^{* a}$, Krystsina Husak ${ }^{a b}$, Ihar Zhuk ${ }^{a c}$, Anastasiya Safronava ${ }^{a d}$, \\ Andrei Patapenka ${ }^{a e}$, Vladimir Voronko ${ }^{b f}$, Vladimir Sotnikov $^{b g}$, Maryna Artiushenko $^{b h}$, \\ Anton Baldin $^{c i}$, Sergey Tyutyunnikov ${ }^{c j}$, Mihaela Paraipan ${ }^{c k}$, Anatoliy Berlev ${ }^{c l}$, \\ Ivan Kudashkin $^{c m}$, S.R.Hashemi-Nezhad ${ }^{d n}$ \\ a Joint Institute for Power and Nuclear Research - Sosny, NAS of Belarus \\ Minsk, Krassin str.,99, Republic of Belarus \\ ${ }^{b}$ National Science Center "Kharkov Institute of Physics and Technology", NAS Ukraine \\ Kharkov, Akademicheskaya str., 1, Ukraine \\ ${ }^{c}$ Joint Institute for Nuclear Research, Dubna, Joliot-Curie str., 6, Russia \\ ${ }^{d}$ School of Physics, University of Sydney, New South Wales 2006, Australia \\ E-mail: o.bukhal@gmail.com, stikrina@mail.ru, zhuk@sosny.bas-net.by, \\ a_safronava@zelenybor.com, a.potapenkodtut.by, \\ voronko@kipt.kharkov.ua, sotnik@kipt.kharkov.ua, \\ art@kipt.kharkov.ua, an.baldin@mail.ru, tsi@sunse.jinr.ru, \\ mihaela_paraipan@yahoo.com, berlev@inr.ru, kudashkin@sunse.jinr.ru, \\ rezahn@bigpond. com
}

\begin{abstract}
Cross sections of spallation reactions of ${ }^{181} \mathrm{Ta},{ }^{197} \mathrm{Au},{ }^{207} \mathrm{~Pb},{ }^{209} \mathrm{Bi},{ }^{232} \mathrm{Th},{ }^{238} \mathrm{U}$ heavy metals induced by deuterons with energy of 2, 2.94, 3.5 GeV were determined. The deuteron beams were extracted from the Nuclotron accelerator at the Laboratory of High Energy Physics, JINR, Dubna, Russia. Spallation reactions cross sections were measured using solid state nuclear track detectors (SSNTD) and activation gamma-spectroscopy. Due to the lack of cross sections data in the range of high energies the experimental results could be added in the international database.
\end{abstract}

XXII International Baldin Seminar on High Energy Physics Problems,

15-20 September 2014

JINR, Dubna, Russia

\footnotetext{
* Speaker.
} 


\section{Introduction}

Using of Accelerator driven systems (ADS) for purpose of transmutation and energy amplification is discussed with increased interest in recent decades. Perspective source of neutrons with harder spectrum than in a chain fission process in such systems is obtained by spallation reaction. Spallation refers to nuclear reactions which occur when energetic particles with kinetic energies larger than about $100 \mathrm{MeV}$ per nucleon interact with an atomic nucleus - the target nucleus. The initial collision between the incident projectile and the target nucleus leads to a series of direct reactions (intranuclear cascade) whereby individual nucleons or small groups of nucleons are ejected from the nucleus at energies above a few $\mathrm{GeV}$ per nucleon, fragmentation of the nucleus can also occur. After the intranuclear cascade phase of the reaction, the nucleus is left in an excited state. It subsequently relaxes its ground state by "evaporating" nucleons, mostly neutrons [1]. Target material is important criteria for ADS.

Target material has to fulfill a wide range of criteria, and first - good spallation properties high atom number and density. The target could be made of such material as beryllium, tantalum, tungsten, uranium, plutonium or thorium. There is a lack of data of spallation reaction cross section of heavy metal with the energy of bombarding particle above $500 \mathrm{MeV}$.

This paper presents the results of determination of heavy metals spallation reactions cross sections at different energies of deuteron beams. In this paper spallation rections cross sections mean cross sections for high energy fission. Main experimental technique was SSNTD technique. This method allowes to detect the secondary heavy iones in the range of mass numbers from 20 to approximately 140. Light iones with the charge less then 10 will not be registred deu to small linear energy transfer (LET) value. Spallation reactions residual nuclei can not leave the fissionable foil deu to small range and kinetic energy.

\section{Experiment}

The Experiments were carried out using high energy beams of "Nuclotron" accelerator at the Joint Institute for Nuclear Research, Dubna, Russia and were constructed in the framework of the international project "Energy plus Transmutation of Radioactive Waste".

\subsection{Experimental installation}

The experiments represent the irradiation of "Telescope" setup with deuteron beams with energies of 2, 2.94, $3.5 \mathrm{GeV} /$ nucleon. The experiments were carried out in February 2014. The "Telescope" tube is a plexiglass tube $1 \mathrm{~cm}$ height and $6.5 \mathrm{~mm}$ in diameter which were prepared especially with appropriate to the foils size. For each experiment the tube is fulfilled by metallic foils of interest $\left({ }^{181} \mathrm{Ta},{ }^{197} \mathrm{Au},{ }^{207} \mathrm{~Pb},{ }^{209} \mathrm{Bi},{ }^{232} \mathrm{Th},{ }^{238} \mathrm{U}\right)$. The number of beam particles hitting the setup (or total intensity) is obtained using activation $\mathrm{Al}$ monitors with the help of ${ }^{27} \mathrm{Al}(d, 3 p 2 n){ }^{24} \mathrm{Na}$ reaction. Al monitors were placed on the both sides of experimental kit. More detailed description of the total intensity determination technique can be found in [2]. The scheme of the "Telescope" setup is presented on Figure 1.

For every material of interest a sandwich composed of a metallic foil of the fissionable material in close contact with two artificial mica foils from both side, acting as fission track detectors 

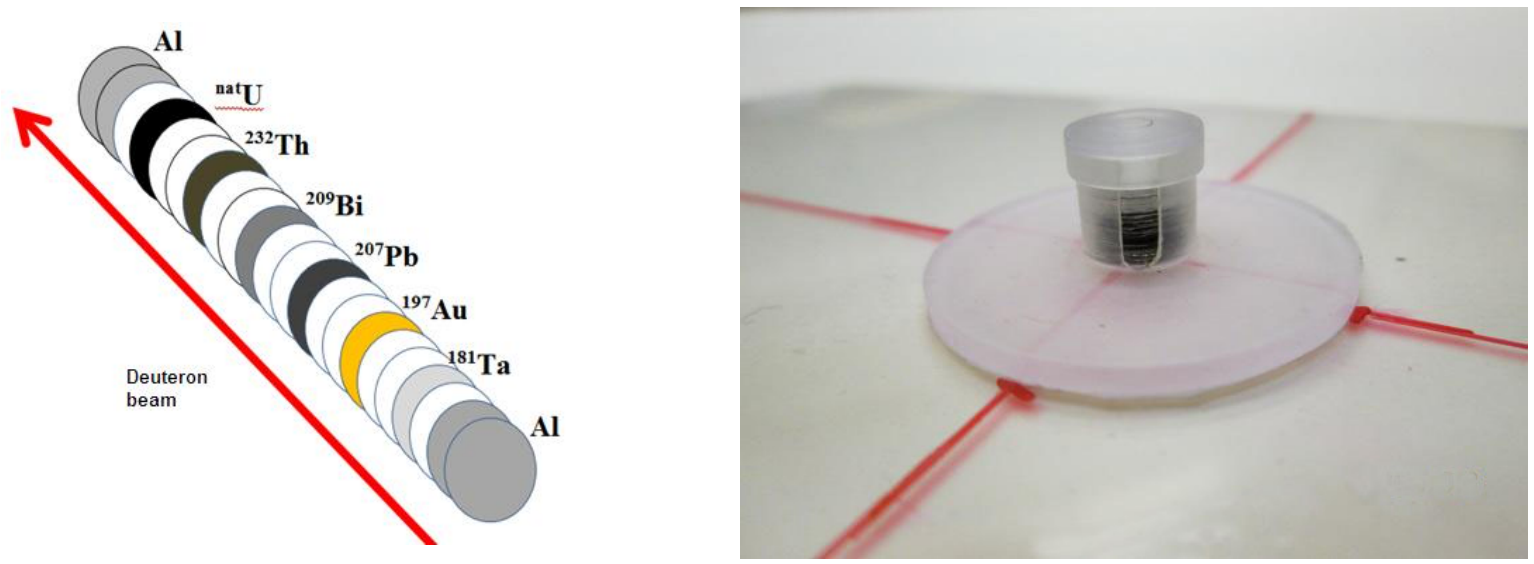

Figure 1: The scheme and the picture of the "Telescope" setup.

\begin{tabular}{|l|c|c|}
\hline Foil of interest & Thickness, $\mathrm{mm}$ & Diameter, $\mathrm{mm}$ \\
\hline $\mathrm{Al}$ & 1.0 & 6.5 \\
${ }^{181} \mathrm{Ta}$ & 0.050 & 6.5 \\
${ }^{197} \mathrm{Au}$ & 0.030 & 6.5 \\
${ }^{207} \mathrm{~Pb}$ & 0.500 & 6.5 \\
${ }^{209} \mathrm{Bi}$ & 0.025 & 6.5 \\
${ }^{232} \mathrm{Th}$ & $0.060-0.080$ & 6.5 \\
${ }^{238} \mathrm{U}$ & 0.030 & 6.5 \\
Mica & 0.030 & 6.5 \\
\hline
\end{tabular}

Table 1: Characteristics of experimental material.

were prepared. All foils were considered to be thick, their thickness $d$ was greater than the range of fission fragments in the foil material that allows o reject an uncertainty of radiator thickness determination. The sizes of investigated heavy metal foils are presented in the Table (1):

Three irradiation by deuterons with energies of 2, 2.94, 3.5 GeV/nucleon were carried out. In each irradiation the "Telescop" setup was mounted on the way of beam at the distance of about $1,5 \mathrm{~m}$ from the beam escape. The investigated setup was placed at the exact beam center after beam focusing with Polaroid film.

Material of detector is synthetic mica (fluorphlogopite). This choice is made taking in account disability of mica to register the recoil nuclei. After the exposure the detectors are etched in $7 \%$ hydrofluoric acid (HF) at $60^{\circ} \mathrm{C}$. The duration of the etching time is decided on the basis of the track population in a given sample, in order to minimize the overlapping of the track openings. After proper etching the tracks are counted by optical microscope.

\subsection{Experimental technique}

To determine reactions cross sections we used solid state nuclear track detectors (SSNTD) and activation gamma-spectroscopy.

SSNTD technique is based on correlation of tracks density on irradiated detectors and flux density of investigated neutron field. After irradiation of track detector in contact with fission foil 
(source of fission fragments) the damaged zone which is called a latent track is formed on the track detector surface. Latent tracks are visible in optical microscope after chemical etching. The conditions of mica etching are mentioned above.

The track density at external surface of a sensor (track detector plus fissionable nuclei) is given by the following equation [3]:

$$
\rho=n \cdot \mu \cdot \varepsilon \cdot d \cdot N_{v} \cdot \int_{0}^{\infty} \sigma_{f}(E) \varphi(E) d E
$$

where $N_{v}$ is number of fissionable nuclei per unit volume of the foil, $\sigma_{f}(E)$ and $\varphi(E)$ are the energy dependent fission cross section and projectile flux respectively, $t$ is the irradiation time, $n$ is number of the fragments emitted per fission, $d$ is the thickness of the foil, $\varepsilon$ is an efficiency factor which include the critical angle effect as well as the limitations imposed by the minimum detectable track size and track observation conditions, $\mu$ is a fraction of charged fragments reached the detector and accounts for different foil thicknesses.

Since using our technique we cannot distinguish what the reaction products leave the tracks on mica high energy fission fragments or other processes fragments, thus under spallation reactions cross section we mean cross sections those reactions which leave the track on the detector surface and understand it more as reactions of high energy fission.

Thereby the equation for spallation reactions cross sections determination is derived from equation (2.1):

$$
\sigma=\rho / P \cdot w
$$

where $P$ is the number of primaries, $w$ is calibration factor. Calibration factor is equal to:

$$
w=n \cdot \mu \cdot \varepsilon \cdot d \cdot N_{v}
$$

Calibration factor depends on the type of fission-foil and on the type of the dielectric detector registering the tracks. Calibration factor is a unique characteristic for specific pair "fissionable foil - detector".

In [4] were discussed the calibration factor in details and have presented experimental and theoretical procedures for its determination [5].

In our calculation we used the calibration factors for ${ }^{181} \mathrm{Ta},{ }^{197} \mathrm{Au},{ }^{207} \mathrm{~Pb},{ }^{209} \mathrm{Bi},{ }^{232} \mathrm{Th},{ }^{238} \mathrm{U}$ which were previously determined in works [5] and are presented in the Table (2):

\begin{tabular}{|c|c|c|c|c|c|c|}
\hline Isotope & ${ }^{181} \mathrm{Ta}$ & ${ }^{197} \mathrm{Au}$ & ${ }^{207} \mathrm{~Pb}$ & ${ }^{209} \mathrm{Bi}$ & ${ }^{232} \mathrm{Th}$ & ${ }^{238} \mathrm{U}$ \\
\hline Calibration factor, $w$ & $0,79 \cdot 10^{19}$ & $1,03 \cdot 10^{19}$ & $1,05 \cdot 10^{19}$ & $0,92 \cdot 10^{19}$ & $0,97 \cdot 10^{19}$ & $0,95 \cdot 10^{19}$ \\
\hline
\end{tabular}

Table 2: Calibration factor for spallation reaction cross sections determination.

\subsection{Result and discussion}

The Table (3) presents the results of the heavy metal spallation reactions cross sections at 2 , 2.94, 3.5 GeV/nucleon deuteron beams measuring.

The uncertainties given in the Table (3) were estimated in accordance with ISO/IEC 17025:1999, ISO/IEC 17025:2001. The budget of uncertainties values for one of investigated foil involved in spallation reactions cross section calculation is presented in the Table (4). 


\begin{tabular}{|c|c|c|c|}
\hline \multirow{2}{*}{ Isotope } & \multicolumn{3}{|c|}{ Cross sections, mbarn } \\
\cline { 2 - 4 } & $2.0 \mathrm{GeV} /$ nucleon & $2.94 \mathrm{GeV} /$ nucleon & $3.5 \mathrm{GeV} /$ nucleon \\
\hline${ }^{181} \mathrm{Ta}$ & $250 \pm 70$ & $230 \pm 60$ & $240 \pm 60$ \\
${ }^{197} \mathrm{Au}$ & $240 \pm 70$ & $230 \pm 60$ & $240 \pm 70$ \\
${ }^{207} \mathrm{~Pb}$ & $270 \pm 80$ & $270 \pm 80$ & $260 \pm 70$ \\
${ }^{209} \mathrm{Bi}$ & $410 \pm 120$ & $390 \pm 110$ & $380 \pm 110$ \\
${ }^{232} \mathrm{Th}$ & $1130 \pm 310$ & $980 \pm 280$ & $1040 \pm 300$ \\
${ }^{238} \mathrm{U}$ & $1500 \pm 400$ & $1280 \pm 400$ & $1240 \pm 350$ \\
\hline
\end{tabular}

Table 3: Spallation reaction cross sections of heavy metal.

\begin{tabular}{|l|c|c|c|}
\hline \multicolumn{1}{|c|}{ Value $X_{i}$} & Estimation $x_{i}$ & $\begin{array}{c}\text { Relative standard } \\
\text { uncertainty } u\left(X_{i}\right), \\
\% / \text { percentage } \\
\text { contribution }\end{array}$ & $\begin{array}{c}\text { Type of uncertainty } \\
\text { type of } \\
\text { probability } \\
\text { distribution }\end{array}$ \\
\hline $\begin{array}{l}N, \text { average track density } \\
\text { in the center of foil }\end{array}$ & $\begin{array}{c}0.97 \cdot 10^{5} \\
\text { track/cm }\end{array}$ & $3.6 / 14.2$ & $\begin{array}{c}\text { A } \\
\text { normal }\end{array}$ \\
\hline $\begin{array}{l}P, \text { average deuteron } \\
\text { flux density }\end{array}$ & $\begin{array}{c}4.91 \cdot 10^{10} \\
\mathrm{~d} / \mathrm{cm}^{2}\end{array}$ & $12.7 / 50.2$ & $\begin{array}{c}\mathrm{B} \\
\text { rectangular }\end{array}$ \\
\hline$K_{\text {sen }, \text { calculated calibration factor }}$ & $\begin{array}{c}\text { (rack/d.barn } \\
\text { track } / 10^{-5}\end{array}$ & $2.9 / 11.4$ & $\begin{array}{c}\mathrm{B} \\
\text { rectangular }\end{array}$ \\
\hline $\begin{array}{l}K_{f}, \text { correction for average track } \\
\text { density on the full detector surface }\end{array}$ & 1.00 & $4.3 / 17.0$ & $\begin{array}{c}\mathrm{B} \\
\text { rectangular }\end{array}$ \\
\hline$K_{s s}$, correction for projectiles self-shielding & 1.00 & $0.6 / 2.4$ & B rectangular \\
\hline $\begin{array}{l}K_{n}, \text { correction for fission } \\
\text { non-connected with the } d\end{array}$ & 1.00 & $0.6 / 2.4$ & $\begin{array}{c}\mathrm{B} \\
\text { rectangular }\end{array}$ \\
\hline$K_{\text {impur }}$, correction for impurity atoms & 1.00 & $0.6 / 2.4$ & B rectangular \\
\hline
\end{tabular}

Table 4: Uncertainties budget.

The relative extended uncertainty is estimated as $28 \%$ (It is suppose that the probability coverage is equal to $=95 \%$ ). In uncertainties budget the highest contribution gives the average deuteron flux density estimation uncertainty and it is about $50 \%$.

While the proton induced reactions are investigated very good in a wide range of energy there is a real lack of cross sections reactions data induced by deuteron in investigated range of energies in the literature and database. There is only a few appropriate data for comparison through the data included in the Experimental Nuclear Reaction Database (EXFOR) [6], only for $4 \mathrm{GeV}$ deuteron induced reactions.

The Table (5) presents the comparison of obtained results with other authors' similar results. We can observe quite good agreement in the results, only cross section for gold differs much more, and it have not been clear for us yet. For lead, bismuth, thorium and uranium we can see good results. 


\begin{tabular}{|c|c|c|}
\hline Nuclide & $\begin{array}{c}\text { Spallation reaction cross sections, } \\
\text { mbarn (our data) }\end{array}$ & $\begin{array}{c}\text { Total fission cross sections } \\
\text { mbarn (published data) }\end{array}$ \\
\hline${ }^{197} \mathrm{Au}$ & $240 \pm 70$ & $92 \pm 23[7]$ \\
${ }^{207} \mathrm{~Pb}$ & $270 \pm 80$ & $173 \pm 40[8]$ \\
${ }^{209} \mathrm{Bi}$ & $410 \pm 120$ & $306 \pm 40[8]$ \\
& & $206 \pm 46[7]$ \\
${ }^{232} \mathrm{Th}$ & $1130 \pm 310$ & $1153 \pm 198[7]$ \\
${ }^{238} \mathrm{U}$ & $1500 \pm 400$ & $1453 \pm 350[7]$ \\
\hline
\end{tabular}

Table 5: Comparison of the obtained results, $4 \mathrm{GeV}$ deuteron induced reaction cross sections.

There is no opportunity to compare results obtained for deuteron induced reactions with energies 5,88 and $7 \mathrm{GeV}$. Our results seemed to be absolutely new in the literature and could supplement the database and are used for further calculation or simulation of spallation targets of ADS.

\section{Conclusion}

Spallation reaction cross sections of heavy metal ${ }^{181} \mathrm{Ta},{ }^{197} \mathrm{Au},{ }^{207} \mathrm{~Pb},{ }^{209} \mathrm{Bi},{ }^{232} \mathrm{Th},{ }^{238} \mathrm{U}$ at energy 2, 2.94, $3 \mathrm{GeV} /$ nucleon deuterons beams have determined. Analyzing obtained results we can conclude that heavy metal spallation reactions cross sections strongly depend on atomic number and cross sections changed slightly in the investigated range of energies.

Due to absence of investigated reactions cross sections in literature and our results are absolutely new, our data should be added in the Experimental Nuclear Reaction Database (EXFOR) that allow the scientists dealt with the ADS research to use them in further calculation and simulation.

\section{References}

[1] Accelerator Driven Systems: Energy generation and transmutation of nuclear waste. Status report, IAEA, Vienna, 1997. - $479 \mathrm{p}$.

[2] A.A. Safronava et al., Monitoring of GeV deuteron beam parameters in ADS experiments at the Nuclotron (JINR, Dubna), in proceedings of DIPAC2011 conference, p. 530-532

[3] A.P. Malykhin et al., Measurement of fission density distributions on critical facilities, Vestsi AS BSSR, Ser. Phys-Energ. Navuk, 1970, p. 16-20

[4] S.R. Hashemi-Nezhad et al., MCalibation of track detectors for fission rate determination: an experimental and theoretical study, Nucl.Instr. and Meth.,Vol.A568. P. 816-825

[5] S.R. Hashemi-Nezhad et al., Calibration factors for determination of relativistic particle induced fission rates in ${ }^{\text {nat }} U,{ }^{235} \mathrm{U},{ }^{232} \mathrm{Th},{ }^{\text {nat }} \mathrm{Pb}$ and ${ }^{197} \mathrm{Au}$ foils, Nuclear Instruments and Methods in Physics Research A,664 (2012), p. 154-160

[6] Experimental Nuclear Reaction Data database, https://www-nds.iaea.org/exfor/exfor.htm

[7] S.Stoulos et al., Nuclear fission cross sections induced by deuterons of $4 \mathrm{GeV}$, Physical Review C85,024612 (2012) 
[8] V.V. Sotnikov et al., Experimental determination of the ${ }^{\text {nat }} \mathrm{Pb}(d, f),{ }^{209} \mathrm{Bi}(d, f),{ }^{209} \mathrm{Bi}(d, x n y p)$ nuclear reaction cross sections at $1.6 \mathrm{GeV}$ and $4 \mathrm{GeV}$ deuteron beams, Int.Conf.Cur.Prob.in Nucl.Phys.Atom.Ene, Kyiv, 2010, p 250 